\title{
Correlation between Ultrasound Findings of Ectopic Pregnancy And Operative Findings in Sudanese Women
}

Khairy S Ismail ${ }^{1}$, Aisha A Geilani ${ }^{1}$, Afaq Abdelaziz $^{1}$, Hassan A Elkheir
Mohaya Slfatih A Elaziz Elnour ${ }^{1}$, Kunna $A^{3}$ and Umbeli Taha ${ }^{1}$

${ }^{1}$ Omdorman Islamic university, Department of Obstetrics and gynecology, Africa

${ }^{2}$ Omdorman Maternity Hospital, Central laboratories, Africa

${ }^{3}$ University of Bahri, Department of obstetrics and gynecology, Africa

Received: December 22, 2017; Published: January 11, 2018

*Corresponding author: Akram Elkhier Hassan, Omdorman Maternity Hospital, Central laboratories, Sudan, Africa

Abstract

Designs and objectives: This study is a prospective, descriptive, hospital-based study, done in Omdurman Maternity Hospital and Omdurman New Hospital, to assess correlation between ultrasound findings of ectopic pregnancy and operative findings in Sudanese women.

Methods: The study was conducted on patients attended to gynecological Casualty with vaginal bleeding and abdominal pain in early pregnancy diagnosed as Ectopic pregnancy, ultrasound done to all the patients, with direct interview using predesigned questionnaire, and all of these is after consent obtained, ultrasonic findings was correlated to the intraoperative findings, sensitivity of the ultrasound was calculated using SPSS.

Results: out of 110 women, the most common age was 20-30 years (48.2\%), multiparous were predominated by (53.6\%). The commonest risk factors included; history of IUCD usage found in (52.7\%), history of PID in (29.1\%), history of abdomino-pelvic surgery in (24.5\%), and history of ectopic pregnancy in (6.4\%). On examination, there were $(71.8 \%)$ women were stable, abdominal tenderness found in $(54.5 \%)$, pallor in (57.3\%), and (10\%) of women were shocked. Sensitivity of U/S in diagnosis of ectopic pregnancy was $97.3 \%$.

Conclusion: It was concluded that, $\mathrm{U} / \mathrm{S}$ is reliable tool for diagnosing ectopic pregnancy

Keywords: Ectopic Pregnancy; Ultrasound Intraoperative; Sudan

Abbreviations: TVUS: Transvaginal Ultrasound; TAUS: Transabdominal Ultrasound; SPPS: Statistical Package for Social Science

\section{Introduction}

Implantation of pregnancy outside the normal endometrial cavity called an ectopic pregnancy, which accounts for about $10 \%$ of all pregnancy-related deaths, despite improved diagnostic methods leading to earlier detection and effective treatment [1]. Also, it increases the chances of secondary infertility as well as incidence of the subsequent ectopic pregnancy [2]. The percentages of the women with ectopic pregnancy go to an emergency department with first trimester bleeding, pain, or both about (6 to 16\%) [3]. Which is strongly associated with an increased incidence of pelvic inflammatory disease [4]. Symptoms may present in both ruptured and enraptured cases [5]. Ectopic pregnancy should be suspected in any woman of reproductive age with these symptoms, especially those who have risk factors for an extrauterine pregnancy [6]. The diagnosis is usually made clinically, based upon results of the imaging studies (ultrasound) and laboratory tests (hCG). The diagnosis can also be made by observation of the ectopic gestation at surgery or histopathological examination [7]. Transvaginal ultrasound (TVUS) is the principle approach used for sonographic evaluation of pregnancy of unknown location. TVUS allows for earlier and more reliable detection of an intrauterine or ectopic pregnancy (abdominal pregnancies are an exception) and for more reliable detection of a fetal heartbeat compared with transabdominal ultrasound (TAUS) [7]. A pseudosac can be seen in up to 20 percent of women with an ectopic pregnancy [8]. An extraovarian adnexal mass, seen in 89 to 100 percent of women with ectopic pregnancy, is the most common US finding in tubal pregnancy [8-10].

\section{Methodology}

This is a descriptive, prospective hospital-based study .It was conducted in Omdurman Maternity Hospital and Omdurman New Hospital. Sudan, during the period from August 2014 to Jan 
2015 all women diagnosed clinically and by ultrasound with ectopic pregnancy attended study area were included in the study. Time-frame sample size was be taken in a period of six months from August 2014 to Jan 2015. Variables assessed were: sociodemographic data, previous ectopic pregnancy, contraceptive usage, tubal pathology and surgery, PID, clinical presentation, ultrasound findings and intra-operative findings. Intra-operative findings were correlated with clinical presentation, examination, and ultrasound findings. Sensitivity and specificity was calculated. The data was collected by direct interview using predesigned questionnaire. Abdominal ultrasound, and/or vaginal ultrasound were done to all the patients according to clinical presentation. Ultrasound was done by senior obstetrician and radiologist. The data was analyzed by computer program; statistical package for social science (SPPS), results presented in tables and graphs. The test of significance was be calculated by $\mathrm{P}$ value (0.05: 95\% confidence). Written consent was obtained from SMSB provided to the hospital administration. Women consent was obtained verbally. Privacy of data collected was considered.

\section{Results}

The study included 110 women diagnosed as ectopic pregnancy by ultrasound and clinically and they attended to $\mathrm{OMH}$ and $\mathrm{ONH}$ during the period from Aug. 2014 to Jan. 2015. The study aimed to correlate clinical presentation, and ultrasound findings versus intraoperative findings. Age distribution showed that, 53(48.2\%) of women in the age group of $20-30$ years, $45(40.9 \%)$ in the age group of 31-40 years, $9(8.2 \%)$ in the age group of 19 years or less, other socio demographic distribution of women in the study shown in Table 1. Obstetric history showed that, 58(52.7\%) of women found to have history of IUCD usage, 32(29.1\%) had history of PID, $27(24.5 \%)$ had history of abdominal-pelvic surgery, $7(6.4 \%)$ had history of ectopic pregnancy and 40 one woman had subfertility. No woman found to have IVF as shown in Figure 1. In addition to clinical examination, transvaginal scan for confirmation of the diagnosis was used in 74(67.3\%) of women, and transabdominal scan was used in $(32.7 \%)$ of women. Operators were distributed to $51(46.4 \%)$ radiologists and $59(53.6 \%)$ consultants as shown in Figures $2 \& 3$.

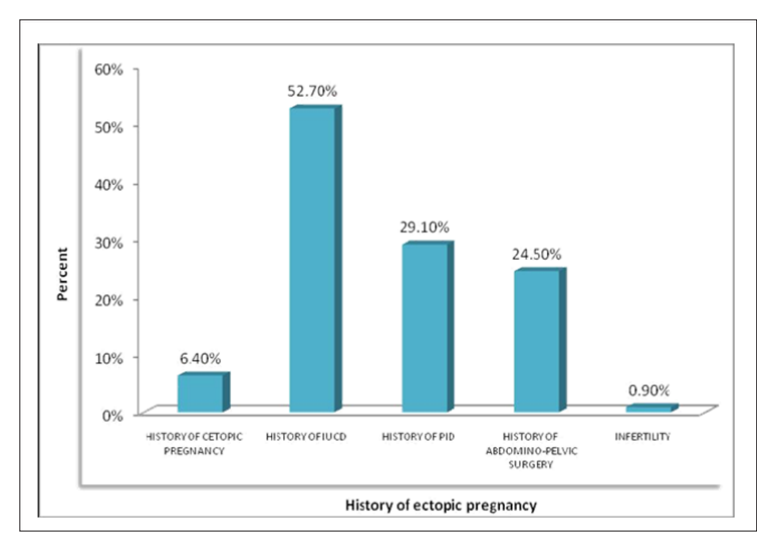

Figure 1: Risk factors distribution among Sudanese women diagnosed with ectopic pregnancy at $\mathrm{OMH}$ and ONH, Aug. 2014 - Jan. 2015

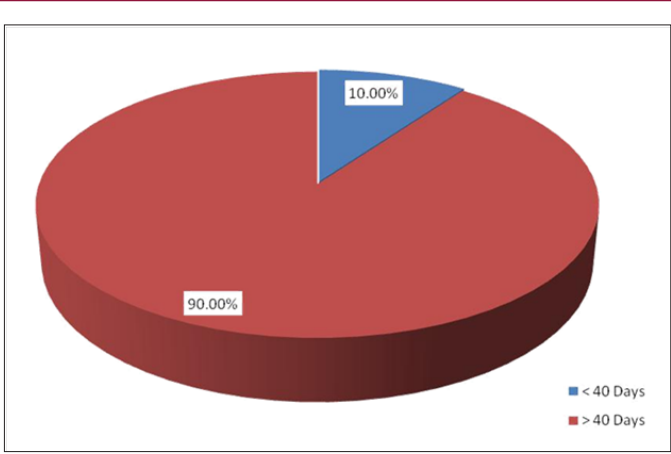

Figure 2: Amenorrhea in Sudanese women diagnosed with ectopic pregnancy at $\mathrm{OMH}$ and $\mathrm{ONH}$, Aug. 2014 Jan. 2015.

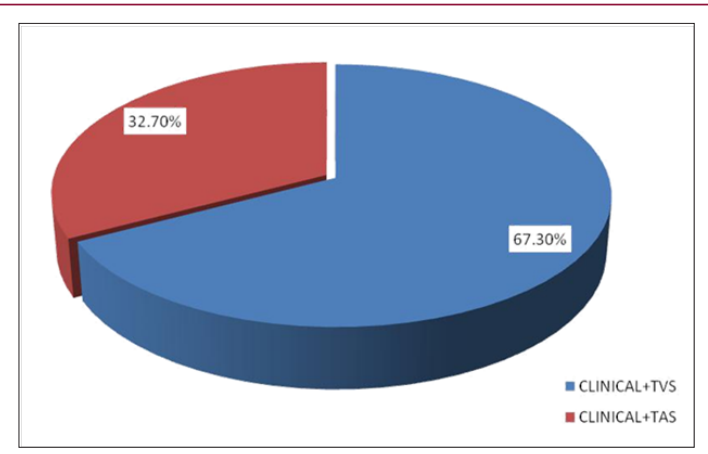

Figure 3: Method of diagnosing Sudanese women with ectopic pregnancy at $\mathrm{OMH}$ and $\mathrm{ONH}$, Aug. 2014 - Jan. 2015.

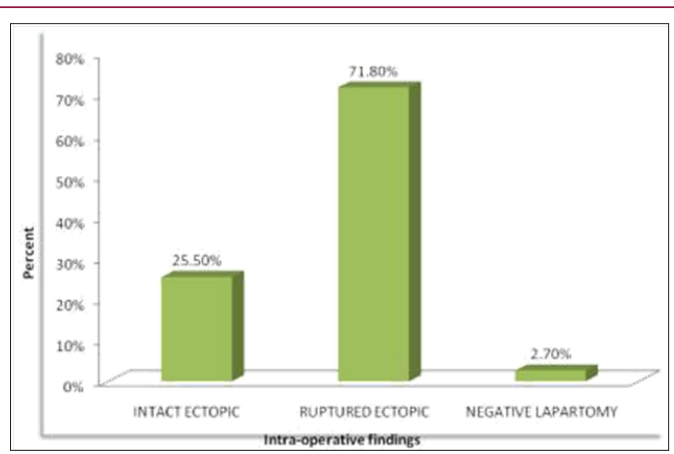

Figure 4: Intra-operative findings in Sudanese women diagnosed with ectopic pregnancy at $\mathrm{OMH}$ and $\mathrm{ONH}$, Aug. 2014 - Jan. 2015

Table 1.

\begin{tabular}{|c|c|c|c|}
\hline \multicolumn{2}{|c|}{ Demography } & Frequency & Percent \\
\hline \multirow{4}{*}{ Age } & $>19$ & 9 & $8.2 \%$ \\
\cline { 2 - 4 } & $20-30$ & 53 & 48.2 \\
\cline { 2 - 4 } & $31-40$ & 45 & 40.9 \\
\cline { 2 - 4 } & $>40$ & 3 & 2.7 \\
\hline \multirow{3}{*}{ Occupation } & Housewife & 83 & 75.5 \\
\cline { 2 - 4 } & Employer & 25 & 22.7 \\
\cline { 2 - 4 } & Labor & 2 & 1.8 \\
\hline Residence & Rural & 18 & 16.4 \\
\hline
\end{tabular}




\begin{tabular}{|c|c|c|c|}
\hline \multirow{4}{*}{ Education } & Urban & 92 & 83.6 \\
\hline & Illitrate & 5 & 4.5 \\
\cline { 2 - 4 } & Primary & 36 & 32.7 \\
\cline { 2 - 4 } & & 38 & 34.5 \\
\cline { 2 - 4 } & Secondary & 31 & 28.2 \\
\cline { 2 - 4 } & University & & \\
\hline \multirow{4}{*}{ Parity } & Primigravida & 15 & 13.6 \\
\cline { 2 - 4 } & Multigravida & 59 & 53.6 \\
\hline & Grandmultipara & 36 & 32.7 \\
\hline
\end{tabular}

Intra-operative findings showed intact ectopic pregnancy in $28(25.5 \%)$, ruptured ectopic was found in $79(71.8 \%)$ and negative laparotomy found in $3(2.7 \%)$ as shown in Figures 4 \& 5. Specific findings showed that gestational with positive fetal cardiac activity in $10(9.1 \%)$ of women, gestational sac containing fetal pole and/or yolk sac in 13(11.8\%). Non-specific findings of ultrasound imaging showed complex mass in $3(2.7 \%)$, complex mass with free fluid in 54(49.1\%), free abdominal fluid in $0(9.1 \%)$ and pre-pelvic + complex mass with free fluid in $20(18.2 \%)$. All participants found managed surgically by laparotomy. Correlation between intraoperative and ultrasound findings showed significant association $(\mathrm{P}$ value $=0.002), 19(67.9 \%$ out of 28 specific findings $)$ of women with intact ectopic showed specific finings in $\mathrm{U} / \mathrm{S}$, among women with ruptured ectopic (79), 76(96.2\%) had non-specific U/S findings, while all women with negative laparotomy had non-specific U/S findings. Correlating intra-operative findings with tender abdomen and shocked patients showed no significant association ( $\mathrm{P}$ value $>$ 0.05).

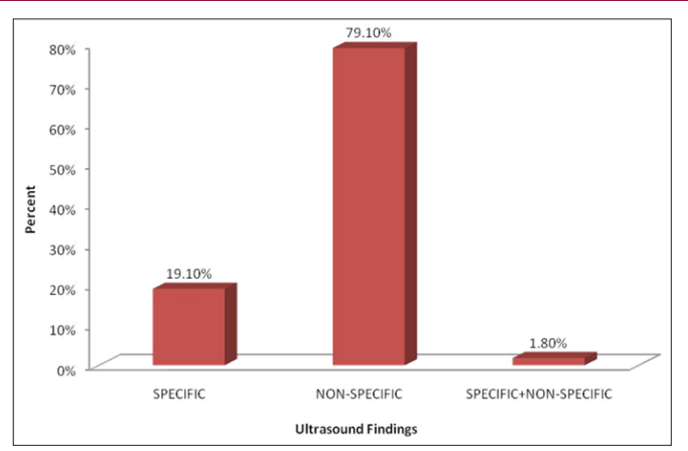

Figure 5: Ultrasound findings in Sudanese women diagnosed with ectopic pregnancy at $\mathrm{OMH}$ and $\mathrm{ONH}$, Aug. 2014 - Jan. 2015

\section{Discussion}

This study carried out in $\mathrm{OMH}$ and $\mathrm{ONH}$ to assess correlation between ultrasound findings of ectopic pregnancy and operative findings in Sudanese women total number of patients was 110 , age distribution showed that, 53(48.2\%) of women had age of 20 30 years, 45(40.9\%) had age of 31-40 years, 9(8.2\%) had age 19 years and less. Multiparous women predominated among women in the current study, they represented by (53.6\%), on the other hand findings indicated considerable percentage of women who were grand multiparous (32.7\%) versus few primigravida (13.6\%). Multi-parity revealed by Kopani $\mathrm{F}$ and colleagues as risk factors for ectopic pregnancy 56. The most frequent criteria considered as risk factors for ectopic pregnancy in the current study were history of using intra-uterine contraceptive device, history of pelvic inflammatory disease and history of abdomino-pelvic surgery (previous caesarean section) and they were represented by $52.7 \%, 29.1 \%$ and $24.5 \%$ respectively. Other risk factors with less incidence were history of ectopic pregnancy (6.4\%) and infertility. Similar findings were reported by Parashi $\mathrm{S}$ and colleagues in 2014, who revealed significant association between prior ectopic pregnancy, prior tubal ligation, use of intrauterine device, and prior abdominal/pelvic surgery with ectopic pregnancy (p<0.05) 57 (12).

Of the risk factors reported by Kopani F and colleagues, there was previous surgery was registered in $26 \%$ of patients and history of previous ectopic pregnancy in $7-8 \%$ of patients reported high incidence of ectopic pregnancy [5-7]. While other studies vast majority of women in the current study presented with vaginal bleeding and abdominal pain (86.4\%), while abdominal pain was found in $13.6 \%$ women. These findings are compatible with what reported by Parashi S and colleagues [8] in 2014 who found that, initial symptoms were nonspecific, consisting of a period of amenorrhea and abdominal pain or tenderness, with or without unexpected vaginal bleeding. New onset pain was reported, dull or sharp in nature, which was generalized or localized to one area. Furthermore, they complained about spotty or irregular vaginal bleeding. Other study reported that, abdominal pain was a presenting symptom in 99 percent, amenorrhea in 74 percent, and vaginal bleeding in 56 percent. On examination at presentation, most patients found stable, pale and have abdominal tenderness which represented by $71.8 \%, 57.3 \%$ and $54.5 \%$ respectively. Shock was found in $10 \%$, and no soft abdomen noticed. In absence of ultrasound findings, HCG test, clinical manifestation of ectopic pregnancy complicates the diagnosis because of their broad spectrum that run from asymptomatic until acute abdomen and hemodynamic shock.

Most women diagnosed through transvaginal scan (67.3\%), less women diagnosed by transabdominal scan. Kopani F and colleagues revealed that, trans-vaginal ultrasound has a sensitivity of $97 \%$ and specificity of $95 \%$. The most frequent intra-operative findings of the current study was ruptured ectopic (71.8\%), followed by intact ectopic which found in $25.55 \%$ and negative laparotomy in $2.7 \%$. Intra-operative findings were reported by Tulandi $\mathrm{T}$ showed that, in one representative series of 147 patients with ectopic pregnancy (78 percent were ruptured) [8]. On the other hand, most findings by ultrasound diagnosis were non-specific (79.1\%) (E.g. Complex mass with free fluid and free abdominal fluid), while specific findings reported in $19.1 \%$ (e.g. Gestational sac containing fetal pole and/or yolk sac). An extra ovarian adnexal mass was reported in previous study as most common non-specific sonographic findings; they reported that, it was seen in 89 to 100 percent of women with ectopic pregnancy, is the most common US finding in tubal pregnancy $(20,26)$. The sensitivity of U/S method in the current study was found to be $97.3 \%$. Findings of the study revealed good accuracy of ultrasound method to diagnose ectopic pregnancy, because imaging was mostly done by seniors and any 
suspicious result repeated several times in other place.

Out of 110 cases suspected with ectopic pregnancy with U/S method, only 3 cases found negative. Of the limitations faced by the current study, is scarcity of published literature comparing U/S imaging and intra- operative diagnosis in ectopic pregnancy patients, the only study available conducted in London by Condous G and colleagues in 2005, it assessed accuracy of TVS accuracy, and it was concluded that, the sensitivity of TVS found to be $90.9 \%$. All women in the current study managed surgically by laparotomy, and they were all ectopic, salpingectomy was done. Intra-operative findings indicated that, intact ectopic were 28, out of which $67.9 \%$ showed specific findings in U/S. out of 79 ruptured ectopic, most cases showed non-specific findings in U/S. while all negative laparotomy showed non-Specific findings. Intra-operative findings indicated that, all shocked women in the study were found ruptured ectopic, except one case which was found intact. Correlating intra-operative findings with shocked cases and with abdomen tenderness found statistically not significant ( $P$ value was 0.326 and 0.230 respectively).

\section{Conclusion}

According to the current findings, we can conclude that, assessing the role of ultrasound in diagnosing ectopic pregnancy in comparison to intra-operative findings, high diagnostic accuracy was revealed with sensitivity of $97.3 \%$. The study obtained good results because imaging was mostly done by seniors and any suspicious result repeated several times in other place. With regard to clinical presentation, most patients were stable, pale and have abdominal tenderness in addition to that some patients were in shocked. From the study the risk factors for ectopic pregnancy were history of using intra-uterine contraceptive device, history of pelvic inflammatory disease and history of abdominal-pelvic surgery. The most common intra-operative findings of the current study was ruptured ectopic, followed by intact ectopic and negative laparotomy.

\section{Recommendations}

a) Since ectopic pregnancy is a top emergency situation, and it is a leading cause of maternal death, clinical assessment should be done as accurate as possible, and cases should be scanned by highly skilled cadres. b) Establishment of early pregnancy assessment unit in every maternity hospital.

c) Organization of IUCD workshops including proper treatment of associated infection.

d) All hospitals, especially obstetrical department should be supplied by well-established U/S unit and well trained operators.

e) Availability of laparoscopic unit with experienced personnel for managing intact ectopic and stable patients laparoscopically.

f) Ultrasound and intra-operative findings can help establishing a reported program to monitor, evaluate and manage ectopic pregnancy by routine studies.

\section{References}

1. Fylstra DL (1998) Tubal pregnancy: a review of current diagnosis and treatment. Obstet Gynecol Surv 53(5): 320-328.

2. Rivillas F, Gómez JG, Jaramillo D (2001) Embarazo ectópico. Series Pretest medicina Obstetricia y Ginecología. Editor Univ Antioquia 10-12.

3. Murray, Heather, Baakdah, Hanadi, Bardell, et al. (2005) Diagnosis and treatment of ectopic pregnancy. CMAJ 173(8): 905-912.

4. Kamwendo F, Forslin L, Bodin L, Danielsson D (2000) Epidemiology of ectopic pregnancy during a 28 year period and the role of pelvic inflammatory disease. Sex Transm Infect 76(1): 28-32.

5. Tulandi T (2014) Clinical manifestations, diagnosis, and management of ectopic pregnancy. UpToDate.

6. Weckstein LN (1985) Current perspective on ectopic pregnancy. Obstet Gynecol Surv 40(5):259-272.

7. Nyberg DA, Mack LA, Jeffrey RB, Laing FC (1987) Endovaginal sonographic evaluation of ectopic pregnancy: a prospective study. Am J Roentgenol 149(6): 1181-1186.

8. Chiang Gloria, Levine Deborah, Swire Michelle (2004) The intradecidual sign: is it reliable for diagnosis of early intrauterine pregnancy? Am J Roentgenol 183(3): 725-731.

9. Bhatt Shweta, Ghazale Hamad, Dogra VS (2007) Sonographic evaluation of ectopic pregnancy. Radiol Clin North Am 45(3): 549-560.

10. Hertzberg BS, Kliewer MA, Bowie JD (1999) Adnexal ring sign and hemoperitoneum caused by hemorrhagic ovarian cyst: pitfall in the sonographic diagnosis of ectopic pregnancy. AJR Am J Roentgenol 173(5): 1301-1302.

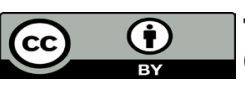

This work is licensed under Creative Commons Attribution 4.0 License

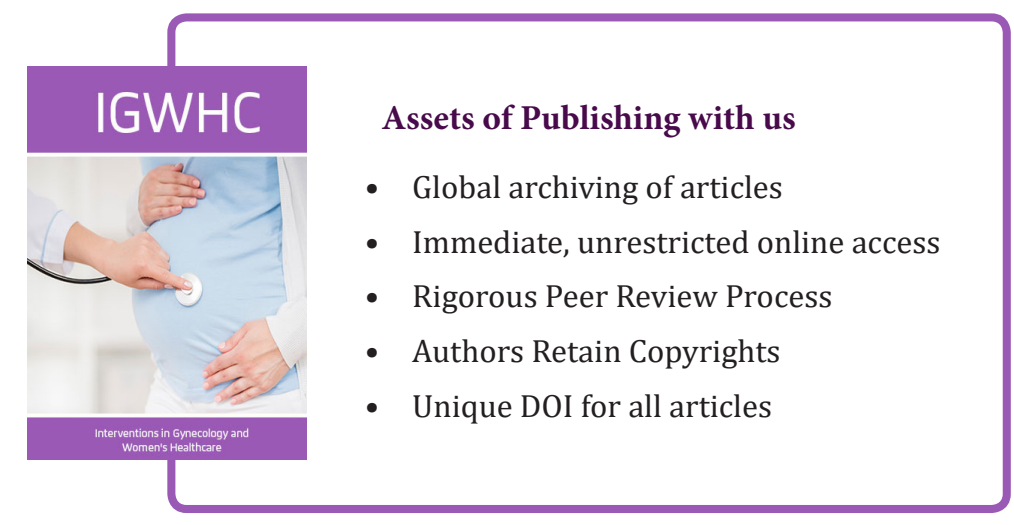

